November 4, 2018

\title{
Top pair Asymmetries at Hadron colliders with general $Z^{\prime}$ couplings
}

\begin{abstract}
Seyed Yaser Ayazi
School of Particles and Accelerators, Institute for Research in

Fundamental Sciences (IPM), P.O. Box 19395-5531, Tehran, Iran

Abstract

Recently it has been shown that measurement of charge asymmetry of top pair production at LHC excludes any flavor violating $Z^{\prime}$ vector gauge boson that could explain Tevatron forward-backward asymmetry (FBA). We consider the general form of a $Z^{\prime}$ gauge boson including left-handed, right-handed vector and tensor couplings to examine FBA and charge asymmetry. To evaluate top pair asymmetries at Tevatron and LHC, we consider $B_{q}^{0}$ mixing constraints on flavor changing $Z^{\prime}$ couplings and show that this model still explain forward-backward asymmetry at Tevatron and charge asymmetry can not exclude it in part of parameters space.
\end{abstract}

\section{Introduction}

The top quark is the only Standard Model (SM) particle which its mass is at the order of the electroweak symmetry breaking and its lifetime is very short. This feature causes that it decays before it can form any hadronic bound state. Thanks to these particular features, careful measurement of top quark properties may be sensitive to new physics (NP).

Experimental results for the cross section of top pair at Tevatron and LHC are well consistent with the SM prediction. While waiting for discovery of NP at the LHC, CDF and D0 collaborations report deviation from SM prediction in the FBA in top pair production [1, 2]. Actually, this observation at Fermilab Tevatron may already be a hint of NP. In the SM, top pair production can be produced via the $q \bar{q}$ annihilation and $g g$-fusion. The 
interference between radiative corrections involving gluon emission and box diagrams lead to FBA in the top pair production [3].

Many extensions of SM have been proposed to explain the measured FBA. Some of these models propose unknown heavy particles which can be exchanged in top pair production process [4]. Possible new particles which can contribute to $t \bar{t}$ production are flavor violating $Z^{\prime}[5], W^{\prime}$ vector boson [6], spin-2 boson [7], axigluons [8], twin Higgs model [9], colored Kaluza Klein excitations of gluon in warped Ads space [10], color-triplet scalar [1] and color-sextet scalar [12.

The LHC allows us to investigate the properties of the top quark in details. A very large number of top quarks are produced at the LHC eventually more than $10^{7} t \bar{t}$ pairs per year [13]. This will make feasible the precise investigations of the top interactions. Since the initial state of proton-proton collisions at the LHC is symmetric, charge asymmetry (CA) manifestly is different from FBA [14]. CA at the LHC is defined as the difference between events with positive and negative absolute values of rapidities of top and antitop quarks. The CMS collaboration has recently presented CA measurement in $t \bar{t}$ production at the LHC for the center of mass energy $7 \mathrm{TeV}$ and $1.09 \mathrm{fm}^{-1}$ of data. This measurement is well consistent with the SM prediction. Nevertheless, the measurement of CA at LHC can provide an independent criterion of NP models which explain FBA at Tevatron.

One of the models which can explain FBA anomaly at Tevatron is flavor violating $Z^{\prime}$ vector boson. Recently, it has been shown [15], that the LHC CA measurement exclude flavor violating $Z^{\prime}$ vector boson which explain the Tevatron FBA. However, In this paper, we consider general form of $Z^{\prime}$ flavor boson which includes right-handed, left-handed vector and tensor terms. We study the effect of this model on observables FBA, CA, and total cross section of $t \bar{t}$ at the Tevatron and LHC. The main point is that coupling of flavor violating $Z^{\prime}$ exchange can contribute to $B_{q}(q=s, d)$ mixing. For 
this reason, to study the impact of $t u Z^{\prime}$ vertex to top pair asymmetry, we will consider $B_{q}$ constraints on these couplings.

The rest of this paper is organized as follows: In the next section, we introduce $Z^{\prime}$ boson with general coupling and its effect on the cross section production of top-antitop. In section 3 we summarize observables which we study at the LHC and Tevatron and study the effects of flavor violating $Z^{\prime}$ boson with general coupling on our observables. The conclusions are given in section 4 .

\section{Flavor Violating $Z^{\prime}$ boson with general coupling}

In this section, we will focus on the model with $Z^{\prime}$ gauge boson that has general coupling and briefly describe the model and phenomenological constraints on its parameters space.

One of the extensions of SM has been proposed to resolve discrepancy of FB asymmetry measured by $\mathrm{CDF}$ and $D 0$ collaborations is the flavor violating $Z^{\prime}$ vector gauge boson. However, recently [15] it has been shown that the measurements of $\mathrm{CA}$ at $\mathrm{LHC}$ have excluded any $Z^{\prime}$ vector gauge boson. In this paper, we consider similar extension of SM with $Z^{\prime}$ gauge boson which has a general coupling including vector, axial vector and tensor coupling. Since a tree level $d b Z^{\prime}$ and $s b Z^{\prime}$ coupling contribute to $B_{d, s}^{0}$, we ignore these terms in Lagrangian. The most general Lagrangian for flavor changing $t q Z^{\prime}(q=u, c, t)$ transition has been given by [16]:

$$
\mathcal{L}_{t u Z^{\prime}}=\bar{u}\left[\gamma^{\mu}\left(a+b \gamma_{5}\right)+i \frac{\sigma_{\mu \nu}}{m_{t}} q^{\nu}\left(c+d \gamma_{5}\right)\right] t Z_{\mu}^{\prime},
$$

where $a, b, c$ and $d$ are real constants and $q=p_{t}-p_{u}$. This Lagrangian can contribute to $t \bar{t}$ production at hadron colliders via t-channel exchange of the $Z^{\prime}$ boson.

This Lagrangian with large flavor changing up type quarks contribute to FCNC processes and $B_{q}^{0}$ mixing. For instance, $t u Z^{\prime}$ coupling will generate 
a $b q^{\prime} Z^{\prime}\left(q^{\prime}=d, s\right)$ coupling at loop level which contribute to $B_{q}^{0}$ mixing. As a result, $B_{q}^{0}$ mixing constrain the $t q^{\prime} Z^{\prime}$ coupling. Constraints on $t q^{\prime} Z^{\prime}$ coupling have been estimated in [17]. To calculate FBA and CA, we consider these constraints on the couplings. Note that right-handed $t u Z^{\prime}$ coupling do not contribute to $B_{q}^{0}$ mixing in the limit that up quark mass set to zero. Also in the limit of low energy, the effect of tensor coupling ( $c$ and $d$ ) on $B_{q}^{0}$ mixing suppressed by $\sim m_{b} / m_{t}$ and consequently, we can ignore the $B_{q}^{0}$ mixing constraints on these couplings. In the following, we introduce top pair production observables at hadron colliders and consider the above coupling effects on them.

\section{Observables and Numerical results}

In this section, we study the total cross section of top pair production at the Tevatron and LHC and consider top pair forward -backward asymmetry and charge asymmetry as observables and study effect of $Z^{\prime}$ gauge boson on them.

Top pair production cross section at Tevatron $(\sqrt{s}=1.96 \mathrm{TeV})$ has been measured by D0 collaboration with $5.4 \mathrm{fb}^{-1}$ of integrated luminosity [18]:

$$
\sigma_{\text {Tevatron }}(p p \rightarrow t \bar{t}) \quad=7.56 \pm 0.83[p b](\text { stat } \oplus \text { sys })
$$

The cross section value for top pair production at LHC have been measured by CMS experiment recently [19]:

$$
\sigma_{\mathrm{LHC}}(p p \rightarrow t \bar{t})=165.8 \pm 13.3[p b](\text { stat } \oplus \text { sys }) .
$$

These measurements are in good agreement with the SM prediction [20, 21]. The tree-level total cross section for $q q^{\prime} \rightarrow t \bar{t}$ including both $\mathrm{SM}$ and $Z^{\prime}$ contribution has been calculated in [17]. The total cross section of top pair production at hadron colliders can be obtained by convoluting the partonic cross section with the parton distribution functions $(\mathrm{PDF})$ for the initial 
hadrons. To calculate $\sigma(p p \rightarrow t \bar{t})$, we have used the CTEQ6L parton structure functions [22] and set the center-of-mass energy to $7 \mathrm{TeV}$. The total cross section for production of $t \bar{t}$ has the following form:

$$
\sigma(p p \rightarrow t \bar{t})=\sum_{a b} \int d x_{1} d x_{2} f_{a}\left(x_{1}, Q^{2}\right) f_{b}\left(x_{2}, Q^{2}\right) \widehat{\sigma}(a b \rightarrow t \bar{t}),
$$

where $f_{a, b}\left(x_{i}, Q^{2}\right)$ are the parton structure functions of proton. $x_{1}$ and $x_{2}$ are the parton momentum fractions and $Q$ is the factorization scale.

Here, we emphasis that for proton-antiproton collision FBA is defined as relative difference between the number of produced top quark with $\cos \theta>0$ and $\cos \theta<0$, which $\theta$ is the production angle in the center of mass system:

$$
A_{F B}=\frac{N_{t}(\cos \theta>0)-N_{t}(\cos \theta<0)}{N_{t}(\cos \theta>0)+N_{t}(\cos \theta<0)}
$$

As it is mentioned, SM model prediction for FBA is as small as a few percent which arises from the interference between the Born amplitude for $q \bar{q} \rightarrow Q \bar{Q}$ and box diagrams and the interference term between initial state radiation and final state radiation $[3$. At the Tevatron, since the initial state is asymmetric (proton-antiproton collisions), the top quark forwardbackward asymmetry can be measured. Recent measurements by CDF [1] $\left(A_{F B}=0.158 \pm 0.075\right)$ and D0[2] $\left(A_{F B}=0.196 \pm 0.065\right)$ collaborations report deviation from the SM prediction which is about $2 \sigma$ larger than the SM (value about 5\%) predictions. At the LHC, initial state is symmetric (proton-proton collisions), as a result FBA vanishes. However, charge asymmetry in $t \bar{t}$ production at LHC can be measured which reflects the top quark rapidity distribution. The top quark charge asymmetry in $t \bar{t}$ is defined by $[?]$

$$
A_{C}=\frac{N_{t}\left(\Delta\left(y^{2}\right)>0\right)-N_{t}\left(\Delta\left(y^{2}\right)<0\right)}{N_{t}\left(\Delta\left(y^{2}\right)\right)>0+N_{t}\left(\Delta\left(y^{2}\right)<0\right)}
$$

where $\Delta\left(y^{2}\right)$ is defined as,

$$
\Delta\left(y^{2}\right)=\left(y_{t}-y_{\bar{t}}\right) \cdot\left(y_{t}+y_{\bar{t}}\right)
$$


and $y_{t}\left(y_{\bar{t}}\right)$ are the rapidity of the top (anti)quark in the laboratory frame. Rapidity difference is a boost invariant observable and is equal to:

$$
y_{t}-y_{\bar{t}}=2 \operatorname{Arctanh}\left(\sqrt{1-\frac{4 \mathrm{~m}_{\mathrm{t}}^{2}}{\hat{\mathrm{s}}}} \cos \theta\right)
$$

while summation of rapidities is not boost invariant and can be written as:

$$
y_{t}+y_{\bar{t}}=\frac{1}{2} \ln \left(\frac{x_{1}}{x_{2}}\right)
$$

In proton-proton collisions at the LHC, the rapidity distributions of the top and antitop quarks are symmetrically distributed around zero. But since the $\mathrm{u}, \mathrm{d}$ valence quarks carry larger average momentum fraction than the anti-quarks, $t \bar{t}$ boost along the direction of the incoming quark, and therefore this leads to a larger average rapidity for top quarks than anti-top quarks. The ATLAS and CMS measurements for the charge asymmetry are: $A_{C}=-0.019 \pm 0.036$ [23], $A_{C}=-0.013 \pm 0.041\left(A_{C}=0.004 \pm 0.014\right)$ [24], and the SM prediction is $A_{C}=0.0115$ [25]. Notice that while measurements of the FBA show a deviation from the SM expectations, measurement of charge asymmetry at the LHC is in agreement with SM prediction. It means that any new physics which explains the $t \bar{t}$ forward-backward asymmetry must satisfy $A_{C}$ measurements consistent with the SM predictions. In the following, we study general form of $Z^{\prime}$ gauge boson and consistency with these measurements.

In numerical calculation, we have set $m_{t}=172.5 \mathrm{GeV}$ and fixed renormalization and factorization scale $\mu_{R}=\mu_{F}=m_{t}$. For including higher order QCD effects, we have normalized all observables to ratio of measured experimental cross section to the leading order SM cross section. In Fig. 1, we have displayed the total cross section of top-antitop production at the Tevatron and LHC as a function of the $Z^{\prime}$ mass. In this figure, $\Gamma_{Z^{\prime}}=2 \mathrm{GeV}$ and different values for couplings are considered. As it is mentioned, due to contribution of $t q Z^{\prime}$ coupling to $B_{q}^{0}$ mixing, the $\Delta M_{q}$ experimental results can 


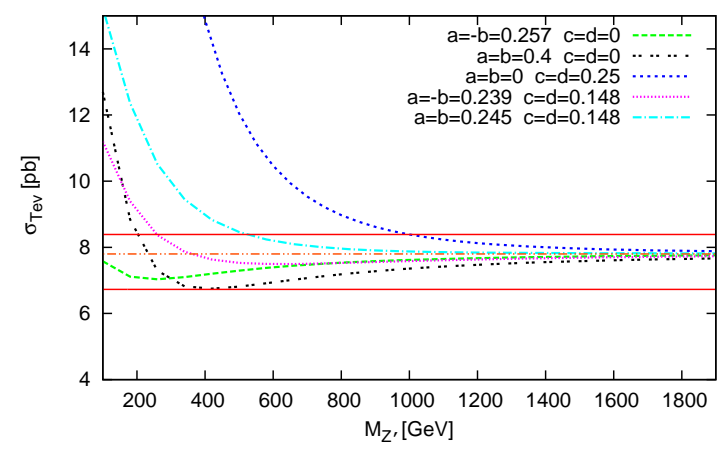

(a)

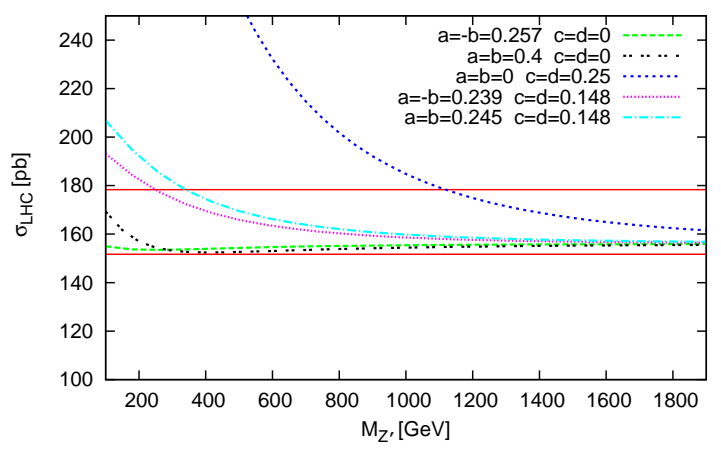

(b)

Figure 1: The top pair production cross section as a function of the $Z^{\prime}$ mass at Tevatron (a) and LHC (b). The horizontal red lines show allowed range of experimental measurements for the top pair total cross section.

constrain these couplings. It is shown [17] that a global analysis on parameters of mass mixing for $B_{d}^{0}$ and $B_{s}^{0}$ mixing constrains $a=-b=g_{L}$ coupling down to 0.4. For $t u Z^{\prime}$ vertex with right-handed coupling $a=b=g_{R}$, we can avoid the $B_{q}^{0}$ mixing due to suppression value $m_{u}^{2} / m_{W}^{2}$. Also for tensor couplings $c$ and $d$, the contribution of these operators to $B_{q}^{0}$ mixing at low energy is negligible [26].

In Fig. 1, we consider all $t u Z^{\prime}$ coupling constraints which arise from $B_{q}^{0}$ mixing. The horizontal red lines show the allowed range of experimental measurement for top pair total cross section at the Tevatron and LHC. The curves with different colors and lines show various values of coupling, according to pseudo vector, vector, tensor and general form of $Z^{\prime}$ couplings.

Fig. 2-a(b) depicts $A_{F B}\left(A_{c}\right)$ at Tevatron (LHC). Different values for couplings have been considered according to $B_{q}$ mixing constraints. As it can be seen, for instance the allowed values for tensor couplings in $A_{F B}\left(A_{C}\right)$ curves are satisfied for $600<M_{Z^{\prime}}<800 \mathrm{GeV}\left(M_{Z^{\prime}}>1500 \mathrm{GeV}\right)$. This means, for given values $a=b=0$ and $c=d=0.25$, there is no allowed region. To better study of all parameters space which simultaneously sat- 


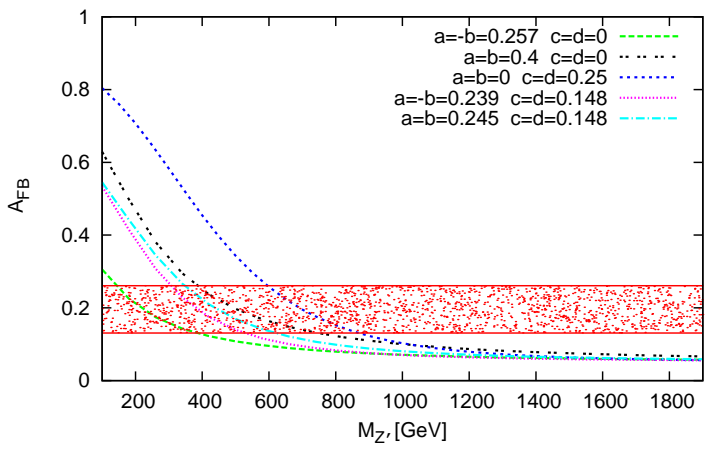

(a)

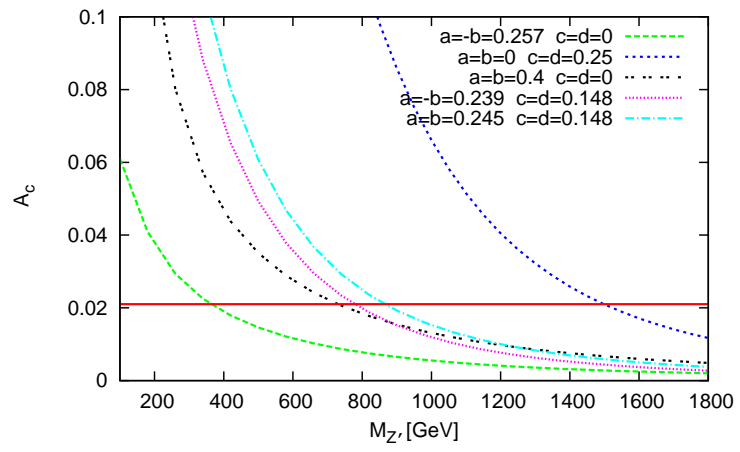

(b)

Figure 2: The Top pair asymmetries as a function of $Z^{\prime}$ mass. a) ForwardBackward asymmetry at Tevatron. b) Charge asymmetry at LHC. The horizontal red lines show the allowed ranges of experimental measurements for asymmetries.

isfies experimental constraints on $\sigma_{\text {Tevatron }}, \sigma_{\mathrm{LHC}}, A_{F B}$ and $A_{C}$, we display Figs. 3 7 and scan parameter space in these categories:

- $Z^{\prime}$ with left-handed and right-handed vector couplings $(c=d=0)$ :

In Fig. 3, shaded areas satisfy experimental measurements of observables $\sigma_{\mathrm{LHC}}, \sigma_{\mathrm{Tev}}, A_{\mathrm{FB}}$ and $A_{\mathrm{C}}$ in (a) right-handed vector and (b) left-handed vector couplings and $m_{Z}$ plane. As it was mentioned, there is no constraints on right handed coupling which come from $B_{q}^{0}$ mixing. As it can be seen, there is no overlapping area between $A_{C}$ and $A_{F B}$ allowable regions for right-handed coupling $\left(a=b=g_{t u}^{R}\right)$. Therefore, measurement of $A_{C}$ at the LHC excludes any $Z^{\prime}$ with right-handed coupling which could explain $A_{F B}$ anomaly at Tevatron.

In Fig. 3-b $\left(a=-b=g_{t u}^{L}\right)$, we also consider $B_{d}^{0}$ mixing constraint on real part of left-handed coupling $\left|g_{t u}^{L}\right|$ which have been taken from Fig. 2 of [17]. For this case, for real value of $g_{L}$, we find regions that all experimental measurement are satisfied. The constraints from $B_{s}^{0}$ mixing on $g_{t u}^{L}$ are suppressed because the contribution of $g_{t u}^{L}$ to $B_{s}^{0}$ mixing is proportional to $V_{u s}^{*} V_{t b}$. 


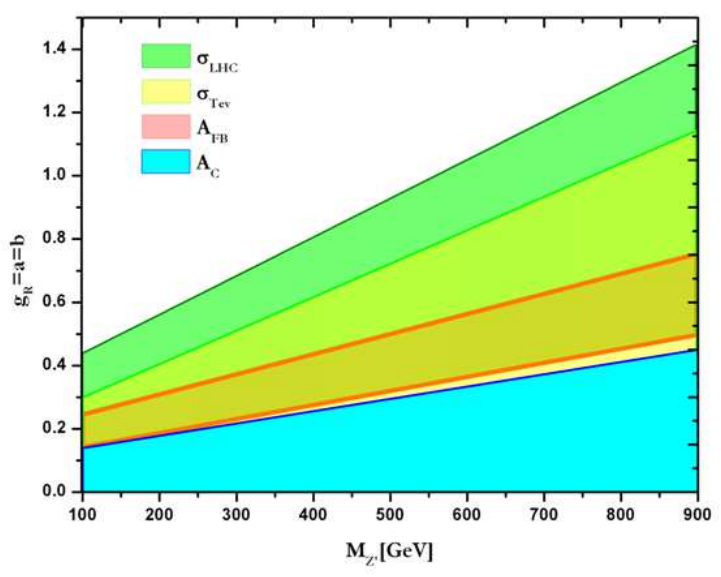

(a)

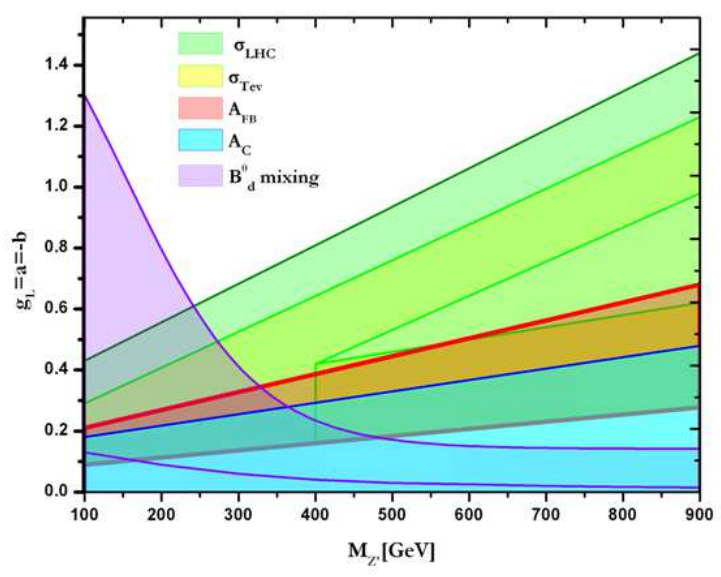

(b)

Figure 3: Shaded areas depict ranges of parameters space in (a) right-handed vector and (b) left-handed vector couplings and $M_{Z^{\prime}}$ plane for which are consistent with experimental measurements and uncertainty of observables: $\sigma_{\mathrm{LHC}}, \sigma_{\mathrm{Tev}}, A_{\mathrm{FB}}, A_{\mathrm{C}}$. Violet area in Fig. (b) shows allowed regions of $\left|g_{t u}^{L}\right|$ which are consistent with experimental $B_{d}^{0}$ mixing constraints.

Nevertheless, $B_{d}^{0}$ mixing strongly constrains the left-handed $t u Z^{\prime}$ coupling. In this paper, we have assumed all couplings are real. In [17, it is shown that for complex coupling $g_{t u}^{L}$, $\operatorname{Arg}\left(g_{\text {tu }}^{L}\right)$ must be between -60 Deg and -20 Deg. Therefore, there are no real $g_{t u}^{L}$ which satisfy the $B_{d}^{0}$ mixing constraints.

- $Z^{\prime}$ with pure tensor couplings $(\mathbf{a}=\mathbf{b}=\mathbf{0})$ : In this case we can neglect the $B_{q}^{0}$ mixing constraints due to suppressed effect of $m_{b} / m_{t}$ at the $b$ mass scale. In Fig. 4, we consider $Z^{\prime}$ with right-handed tensor couplings $(c=d)$. It is notable that allowed regions of top pair production cross section at the LHC and Tevatron, overlap with allowed regions of $A_{C}$ and $A_{F B}$. Nevertheless, there is no overlapping region between the $A_{C}$ measurement at the Tevatron and $A_{F B}$ measurement at the LHC. As a result, $A_{C}$ 
measurement can exclude $Z^{\prime}$ gauge boson with right-handed tensor coupling which could explain $A_{F B}$ anomaly measurement at Tevatron. For the case $c=-d$, the situation is similar to pervious case as expected because cross section is quadratically dependent on $d$ when $b=0$.

For $Z^{\prime}$ with general form of tensor coupling $(0 \leq c \leq 1$ and $|d| \leq$ 1), we display Fig. 5. It is remarkable that there are overlapping regions between measured $A_{C}$ at LHC and measured $A_{F B}$ at Tevatron. This means, flavor changing $Z^{\prime}$ gauge boson with general form of tensor coupling could still explain forward-backward anomaly at Tevatron keeping all observables consistent with measurements. The comparison of Fig. 5ta and Fig. 5-b shows that the allowed region for $Z^{\prime}$ will decline when $Z^{\prime}$ mass increases.

In this figure, we consider situation that $0 \leq c \leq 1$ and $0 \leq d \leq 1$. Similar condition exist for the case $-1 \leq d \leq 0$.

- $Z^{\prime}$ with general form of couplings: Fig. 6 depicts, allowed region of tensor axial and right-handed couplings for which experimental measurements of observables $\sigma_{\mathrm{LHC}}, \sigma_{\mathrm{Tev}}, A_{\mathrm{FB}}$ and $A_{\mathrm{C}}$ are satisfied. As it was mentioned, for right-handed coupling, the contributions of $t u Z^{\prime}$ vertex to $B_{d}^{0}$ and $B_{s}^{0}$ mixing are suppressed by a factor of $m_{u}^{2} / m_{W}^{2}$. As it has been shown in these figures, there are small overlapping regions between the measured $A_{C}$ at the LHC and the measured $A_{F B}$ at the Tevatron. For the case $a=b=1$ and $(c \neq|d|) \leq 1$, we expect that similar to pure tensor coupling, there are allowed regions of $A_{C}$ and $A_{F B}$. In Fig. 7, we consider $Z^{\prime}$ gauge boson with left-handed vector and tensor couplings. For this case, we have taken constraints $B_{d}$ mixing from [17]. Violet area in Fig. 7 shows allowed regions for $\left|g_{t u}^{L}\right|$ which satisfy experimental $B_{d}^{0}$ mixing constraints. In this paper, we suppose $g_{t u}^{L}$ is real and Fig. 77 a shows that for real value of $g_{t u}^{L}$, there is a overlapping region which satisfy all experimental constraints. As it was mentioned (in Fig [3-b) for the case $a=-b=g_{t u}^{L}, B_{d}$ mixing can strongly constrain the $t u Z^{\prime}$ coupling. In fact there are no real $g_{t u}^{L}$ consistent with 


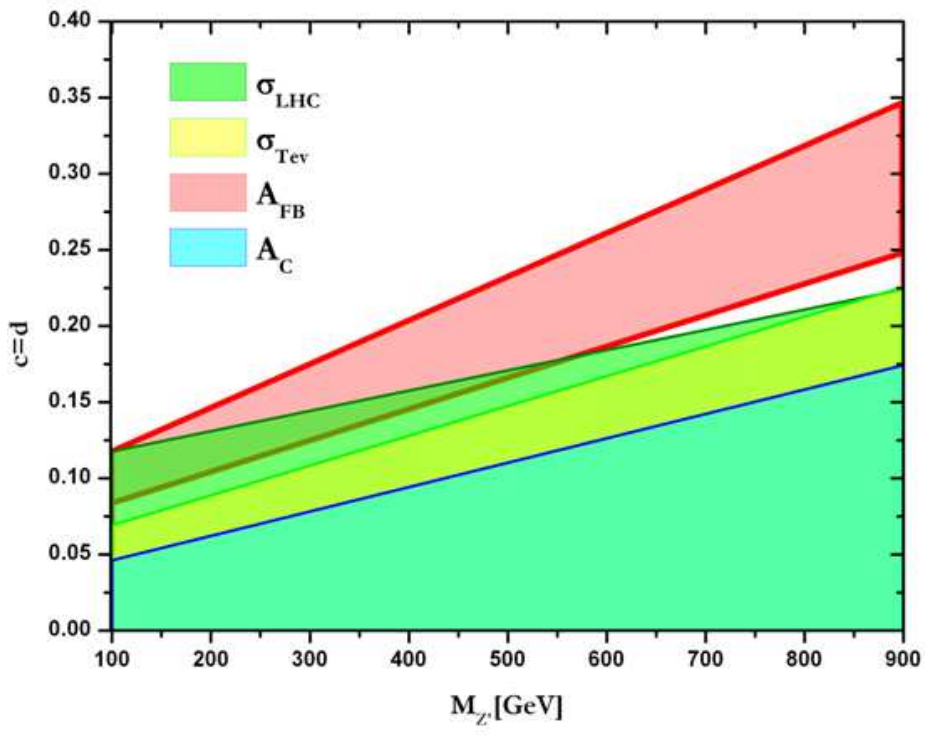

Figure 4: Shaded areas depict ranges of parameters space in right-handed tensor coupling and $M_{Z^{\prime}}$ plane for which are consistent with experimental measurements of observables: $\sigma_{\mathrm{LHC}}, \sigma_{\mathrm{Tev}}, A_{\mathrm{FB}}$ and $A_{\mathrm{C}}$. 


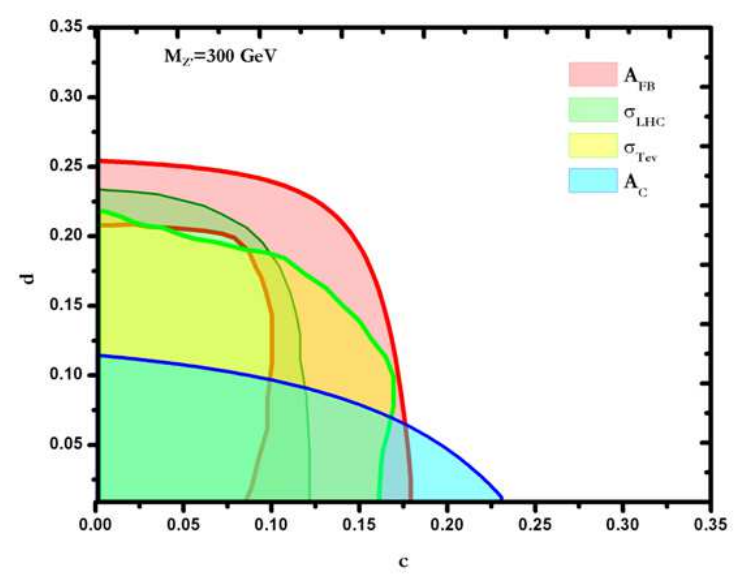

(a)

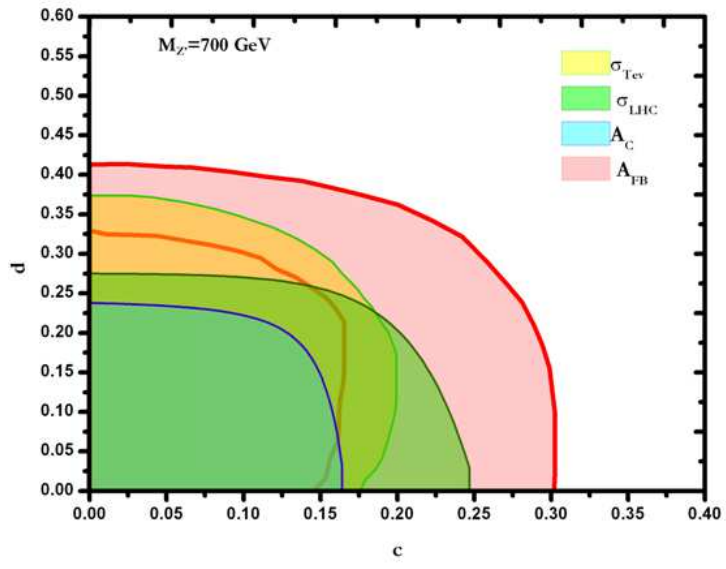

(b)

Figure 5: Shaded areas depict ranges of parameters space in tensor couplings plane for which are consistent with experimental measurements of observables: $\sigma_{\mathrm{LHC}}, \sigma_{\mathrm{Tev}}, A_{\mathrm{FB}}$ and $A_{\mathrm{C}}$. In Fig. (a) $M_{Z^{\prime}}=300$ and in Fig. (b) $M_{Z^{\prime}}=700$. 


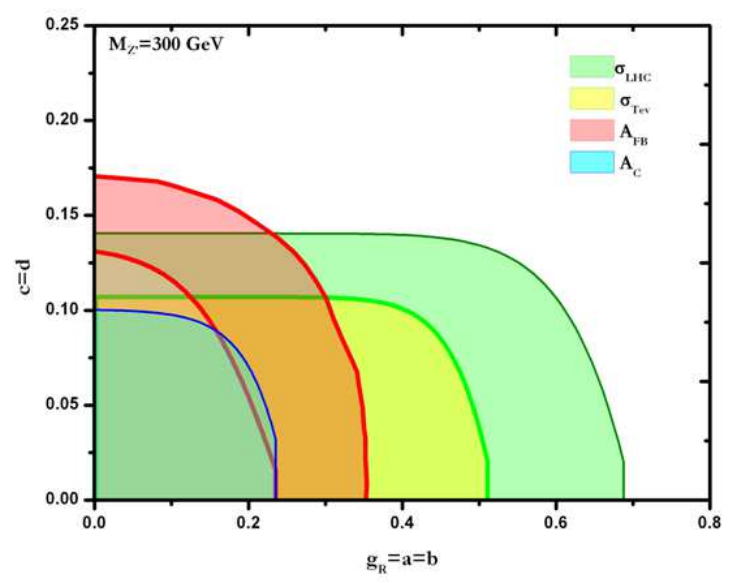

(a)

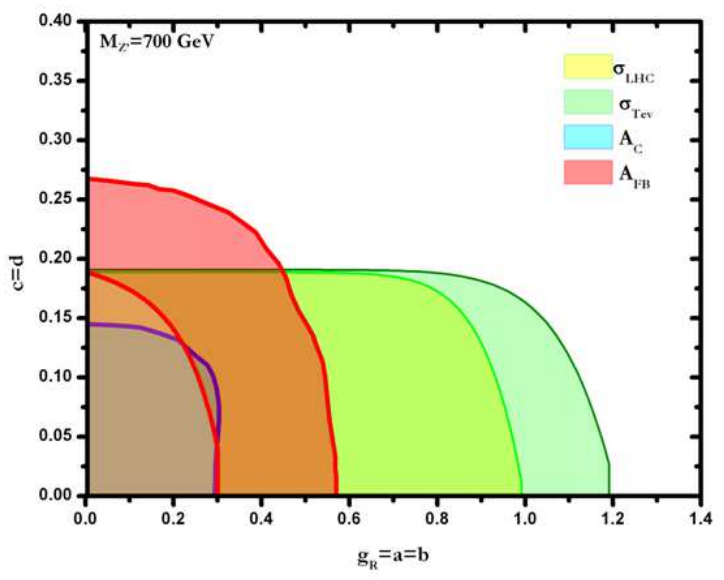

(b)

Figure 6: Shaded areas depict ranges of parameters space in right-handed tensor and vector couplings plane for which are consistent with experimental measurements of observables: $\sigma_{\mathrm{LHC}}, \sigma_{\mathrm{Tev}}, A_{\mathrm{FB}}$ and $A_{\mathrm{C}}$. In Fig. (a) $M_{Z^{\prime}}=$ 300 and in Fig. (b) $M_{Z^{\prime}}=700$

$B_{d}^{0}$ mixing constraints. This means that if we relax $B_{d}^{0}$ constraints on phase of $g_{t u}^{L}$ [17], there are allowed regions for $A_{C}$ and $A_{F B}$ measurements. Nevertheless these values for couplings can not satisfy $B_{d}$ mixing constraints. The case which $a=b=1$ and $(c \neq|d|) \leq 1$ is excluded by experimental $B_{d}^{0}$ mixing constraints.

\section{Concluding remarks}

In this paper, we have studied the effects of $Z^{\prime}$ gauge boson on top pair asymmetries and its cross section productions at the Tevatron and the LHC. It was recently shown that measurement of charge asymmetry of top pair events at the LHC excludes any flavor violating $Z^{\prime}$ vector gauge boson in all of parameters space[15]. We have focused on the flavor changing $Z^{\prime}$ gauge boson model with general form of the couplings including left-handed, right- 


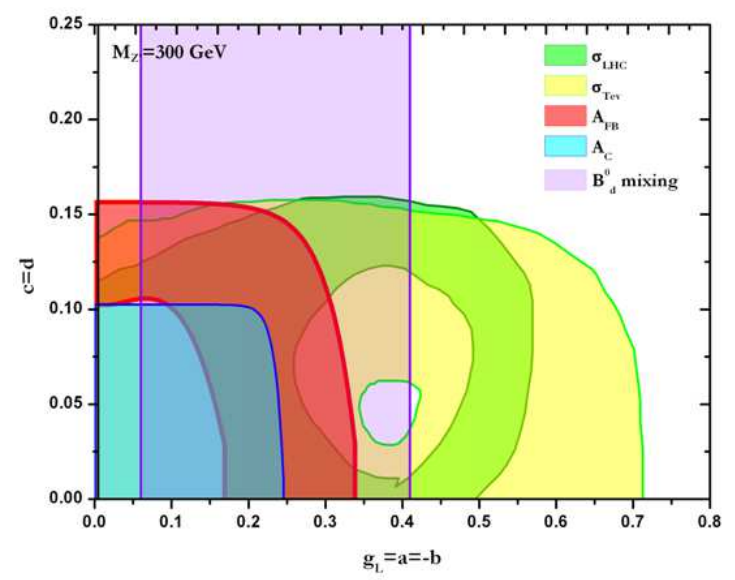

(a)

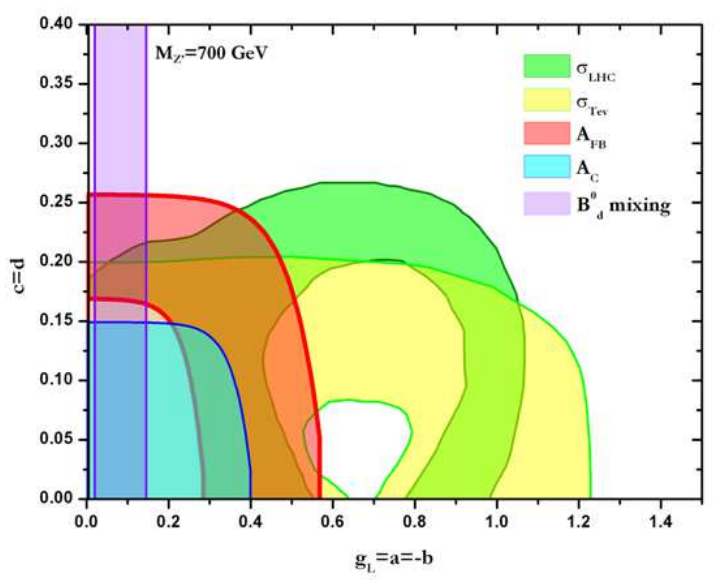

(b)

Figure 7: Shaded areas depict ranges of parameters space in right-handed tensor and left-handed vector couplings plane for which are consistent with experimental measurements of observables: $\sigma_{\mathrm{LHC}}, \sigma_{\mathrm{Tev}}, A_{\mathrm{FB}}$ and $A_{\mathrm{C}}$. Violet area shows allowed regions of $\left|g_{t u}^{L}\right|$ which satisfy experimental $B_{d}^{0}$ mixing constraints. In Fig. (a) $M_{Z^{\prime}}=300$ and in Fig. (b) $M_{Z^{\prime}}=700$. 
handed vector and tensor couplings. We have also discussed the effect of $t u Z^{\prime}$

couplings on $B_{q}^{0}(q=d, s)$ mixing and considered consistent couplings with $B_{d}^{0}$ constraints. We have shown that right-handed vector $t u Z^{\prime}$ coupling, there is no overlapping region which satisfies simultaneously $A_{C}$ and $A_{F}$ measurements. For left-handed couplings, these regions exist but $B_{d}^{0}$ mixing constraint do not allow us to consider these couplings. Similar conditions exist for $t u Z^{\prime}$ left-handed and tensor couplings with specific chirality.

The main point is that if we consider general form of tensor coupling, all experimental measurements including top pair asymmetries and top pair total cross section at the Tevatron and the LHC are satisfied and also $B_{q}^{0}$ mixing does not limit the couplings. For right-handed vector and tensor couplings, there is small region in parameters space which satisfied all experimental bounds. This means flavor changing $Z^{\prime}$ gauge boson still can explain forward-backward anomaly at Tevatron.

\section{Acknowledgement}

The author would like to thank M. Mohammadi Najafabadi for the useful discussions. He is also grateful to S. Paktinat for careful reading of the manuscript and the useful remarks.

\section{References}

[1] T. Aaltonen et al.[CDF Collaboration], Phys. Rev. D83, 112003(2011).

[2] V. Abazov et al. [D0 Collaboration], Phys. Rev. Lett. 84, 112005 (2011), arXiv:1107.4995 [hep-ex].

[3] J. H. Kuhn and G. Rodrigo, Phys. Rev. D 59 (1999) 054017 hep-ph/9807420. 
[4] J. A. Aguilar-Saavedra and M. Perez-Victoria, JHEP 1109 (2011) 097 arXiv:1107.0841 [hep-ph]]; J. A. Aguilar-Saavedra, arXiv:1202.2382 [hep-ph]; P. Ko, arXiv:1202.0367 [hep-ph]; J. A. Aguilar-Saavedra and M. Perez-Victoria, Phys. Rev. D 84 (2011) 115013 arXiv:1105.4606 [hep-ph]]; M. I. Gresham, I. -W. Kim and K. M. Zurek, Phys. Rev. D 85 (2012) 014022 arXiv:1107.4364 [hep-ph]].

[5] S. Jung, H. Murayama, A. Pierce and J. D. Wells, Phys. Rev. D 81 (2010) 015004 [arXiv:0907.4112 [hep-ph]]; M. Frank, A. Hayreter and I. Turan, Phys. Rev. D 84 (2011) 114007 arXiv:1108.0998 [hep-ph]]; J. -Y. Liu, Y. Tang and Y. -L. Wu, J. Phys. G G 39 (2012) 055003 arXiv:1108.5012 [hep-ph]]; M. Endo and S. Iwamoto, arXiv:1110.0014 [hep-ph].

[6] N. Craig, C. Kilic and M. J. Strassler, Phys. Rev. D 84 (2011) 035012 arXiv:1103.2127[hep-ph]]; S. Y. Ayazi, S. Khatibi and M. Mohammadi Najafabadi, arXiv:1205.3311 [hep-ph]; J. Cao, K. Hikasa, L. Wang, L. Wu and J. M. Yang, Phys. Rev. D 85 (2012) 014025 arXiv:1109.6543 [hep-ph]].

[7] B. Grinstein, C. W. Murphy, D. Pirtskhalava and P. Uttayarat, arXiv:1203.2183 [hep-ph].

[8] E. Gabrielli, M. Raidal and A. Racioppi, arXiv:1112.5885 [hep-ph]; E. L. Berger, Q. -H. Cao, C. -R. Chen, J. -H. Yu and H. Zhang, arXiv:1111.3641 [hep-ph].

[9] L. Wang, L. Wu and J. M. Yang, Phys. Rev. D 85 (2012) 075017 arXiv:1111.4771 [hep-ph]].

[10] A. Djouadi, G. Moreau, F. Richard, and R. K. Singh (2009), 0906.0604.

[11] Z. Ligeti, M. Schmaltz and G. M. Tavares, JHEP 1106, 109 (2011); J. Shu, T. M. P. Tait and K. Wang, Phys. Rev. D 81, 034012 (2010); A. 
Arhrib, R. Benbrik and C. H. Chen, Phys. Rev. D 82, 034034 (2010); I. Dorsner, S. Fajfer, J. F. Kamenik and N. Kosnik, Phys. Rev. D 81, 055009 (2010); Phys. Rev. D 82, 094015 (2010).

[12] B. Grinstein , A. L. Kagan, M. Trott and J. Zupan, Phys. Rev. Lett. 107, 012002 (2011);K. M. Patel and P. Sharma, JHEP 1104, 085 (2011).

[13] E. Halkiadakis, arXiv:1004.5564 [hep-ex].

[14] J. H. Kuhn and G. Rodrigo, Phys. Rev. D 59 (1999) 054017 hep-ph/9807420.

[15] S. Fajfer, J. F. Kamenik and B. Melic, arXiv:1205.0264 [hep-ph].

[16] A. Datta and M. Duraisamy, Phys. Rev. D 81, 074008 (2010), arXiv:0912.4785 [hep-ph]].

[17] M. Duraisamy, A. Rashed and A. Datta, Phys. Rev. D 84 (2011) 054018 arXiv:1106.5982 [hep-ph]].

[18] D0 Collaboration, Phys. Lett. B 704, (2011) 403-410.

[19] The CMS Collaboration, CMS-PAS-TOP-11-024.

[20] U. Langenfeld, S. Moch, and P. Uwer , Phys. Rev. D. 80, 054009 (2009), arXiv:0906.5273[hep-ph]]; M. Cacciari et al. , JHEP 09, 127 (2008), arXiv:0804.2800[hep-ph]]; N. Kidonakis and R. Vogt , Phys. Rev. D. 78, 074005 (2008), arXiv:0805.3844 [hep-ph]]; V. Ahrens, A. Ferroglia, M. Neubert, B. D. Pecjak and L. Li , JHEP 09, 097 (2010), arXiv:1003.5827[hep-ph]].

[21] R. Kleiss andW. J. Stirling, Z. Phys. C40 (1988) 419423. doi:10.1007/BF01548856; A. D. Martin,W. J. Stirling, R. S. Thorne et al, Eur. Phys. J. C63 (2009) 189285, arXiv:0901.0002; A. D. Martin,W. J. Stirling, R. S. Thorne et al, Eur. Phys. J. C64 (2009) 653680, 
arXiv:0905.3531; H.-L. Lai, J. Huston, Z. Li et al, Phys.Rev. D82 (2010) 054021, arXiv:1004.4624; F. Demartin, S. Forte, E. Mariani et al, Phys. Rev. D82 (2010) 014002, arXiv:1004.0962.

[22] J. Pumplin, D. R. Stump, J. Huston, H. L. Lai, P. M. Nadolsky and W. K. Tung, JHEP 0207, 012 (2002) arXiv:hep-ph/0201195.

[23] G. Aad et al. [ATLAS Collaboration], arXiv:1203.4211 [hep-ex].

[24] S. Chatrchyan et al. [CMS Collaboration], Phys. Lett. B 709 (2012) 28 arXiv:1112.5100 [hep-ex]]; S. Chatrchyan et al. [CMS Collaboration], Phys. Lett. B 717 (2012) 129 arXiv:1207.0065 [hep-ex]].

[25] [23] J. H. Kuhn and G. Rodrigo, JHEP 1201, 063 (2012).

[26] A. Datta and X. Zhang, Phys. Rev. D 55, 2530 (1997), arXiv:hep-ph/9611247. 